\title{
Tiempo de clampeo del cordón umbilical en recién nacidos de término
}

\author{
Timing of umbilical cord clamping of term infants
}

\author{
Dr. José M. Ceriani Cernadas ${ }^{a, b}$
}

\begin{abstract}
RESUMEN
El tiempo de clampeo del cordón umbilical ocasionó múltiples controversias, al menos, desde hace más de 200 años. En las últimas décadas, predominó marcadamente la práctica del clampeo temprano (primeros 15 segundos). Recién en este siglo, investigaciones aleatorizadas controladas mostraron los beneficios del clampeo demorado del cordón (de 2 a 3 minutos). Esta práctica fue instalada en la asistencia obstétrica por las recomendaciones desociedadescientíficas y delas revisiones sistemáticas, que señalaron las sólidas evidencias que apoyaban esta conducta en neonatos de término.

En esta revisión, se describen los factores más relevantes que sustentan la aplicación del clampeo demorado del cordón en recién nacidos de término. Se destaca el esencial rol que juegan los mecanismos fisiológicos, que, sin duda, nos llevan a comprender los beneficios de esta práctica y nos enseñan a respetar lo que la naturaleza ha determinado. Asimismo, se abordan otros importantes aspectos que también apoyan el empleo del clampeo demorado.

Palabras clave: clampeo, cordón umbilical, transfusión placentaria, transiciónneonatal, deficiencia de hierro.
\end{abstract}

http://dx.doi.org/10.5546/aap.2017.188

Texto completo en inglés:

http://dx.doi.org/10.5546/aap.2017.eng.188

a. Servicio de

Neonatología e

Instituto Universitario,

Hospital Italiano de

Buenos Aires.

b. Consejo de

Publicaciones, Sociedad

Argentina de Pediatría.

Correspondencia:

Dr. José María Ceriani

Cernadas, jose.ceriani@

hospitalitaliano.org.ar

Financiamiento:

Ninguno.

Conflicto de intereses:

Ninguno que declarar.

Recibido: 11-10-2016

Aceptado: 14-10-2016
Cómo citar: Ceriani Cernadas JM. Tiempo de clampeo del cordón umbilical en recién nacidos de término. Arch Argent Pediatr 2017;115(2):188-194.

\section{INTRODUCCIÓN}

Desde mediados del siglo XVIII, el momento de clampeo del cordón umbilical ha sido y sigue siendo un tema de controversias. Erasmus Darwin, distinguido médico y abuelo del famoso Charles, en un libro de su autoría publicado en $1801,{ }^{1}$ se refiere a este aspecto con preocupación: “Otra cosa muy perjudicial para el niño es ligar y cortar el cordón umbilical demasiado pronto, se debe siempre esperar, no solo que tenga respiraciones repetidas, sino hasta que cesen las pulsaciones del cordón. De otra manera se perjudica al recién nacido que es muy débil, ya que una parte de la sangre que queda en la placenta debería haberle correspondido a él".

Pierre Budin, famoso obstetra francés que, además, se dedicó al cuidado de los recién nacidos (RN), es considerado el padre de la neonatología. Publicó, en 1875, un artículo en el que se preguntaba cuál era el momento en que se debía ligar el cordón umbilical. ${ }^{2}$ Este artículo mereció, en 1975, un comentario en Pediatrics con este sugestivo título: “100 años después y todavía no hay respuesta (100 years on and still no answer)". ${ }^{3}$ Nada había cambiado en tantos años.

Luego de la mitad del siglo xx, el clampeo inmediato del cordón fue la práctica generalizada en países occidentales. Varios artículos con escasos pacientes, publicados en las décadas de 1960 y 1970, ${ }^{4-8}$ apoyaron firmemente el clampeo inmediato del cordón, sin ningún sustento de evidencias.

Recién en este siglo, se publicaron estudios bien diseñados que permitieron disminuir las controversias. Las evidencias actuales son lo suficientemente sólidas como para confirmar los innegables beneficios del clampeo demorado del cordón en recién nacidos de término (RNT). ${ }^{9-11}$

Para comprender el porqué de esta afirmación, se describen los principales factores que intervienen en el clampeo demorado en RNT.

\section{Aspectos fisiológicos}

Los mecanismos fisiológicos juegan un rol esencial en los resultados que se 
observan cuando el cordón es pinzado no antes del minuto de vida. De ahí que es esencial conocer adecuadamente cómo funcionan a fin de poder comprender sus efectos sobre el neonato.

\section{Transfusión placentaria}

Es la transferencia de sangre desde la placenta al RN. Constituye un mecanismo fisiológico de trascendental importancia y es la base para entender las diferencias entre los tiempos de clampeo del cordón y sus efectos en el neonato. $\mathrm{Al}$ minuto de vida, el $\mathrm{RN}$ recibe $80 \mathrm{ml}$ de sangre $\mathrm{y}$, entre los dos y los tres minutos, $100 \mathrm{ml}$, lo cual significa alrededor de $30-35 \mathrm{ml} / \mathrm{kg}$, un volumen que es un $75 \%$ más elevado que en el clampeo temprano.

\section{El aporte de hierro durante la transfusión placentaria}

Al nacer, el RNT tiene un depósito de $75 \mathrm{mg} /$ kg de hierro $(\mathrm{Fe}) \mathrm{y}$, mediante la transfusión placentaria con el clampeo demorado, recibe un aporte extra de Fe, $40 \mathrm{mg}$ al minuto y $50 \mathrm{mg}$ a los tres minutos (Figura 1). De esa forma, cuenta con un depósito de Fe de 115 a 125 mg/kg, cantidad que alcanza para prevenir su deficiencia en los primeros 6 meses y, probablemente, hasta el año.

En un estudio que realizamos, ${ }^{12}$ los niveles de ferritina en sangre a los seis meses fueron significativamente más elevados en lactantes que tuvieron clampeo al tercer minuto que en aquellos que lo tuvieron en los primeros segundos. Otros autores hallaron resultados similares. ${ }^{13-23}$

Este efecto tiene enorme importancia futura en los niños porque la deficiencia de Fe en las primeras etapas puede tener efectos perjudiciales en el sistema nervioso central con trastornos cognitivos. Asimismo, es la principal causa de anemia, una afección grave en países en vías de desarrollo y de menor cuantía en los desarrollados. ${ }^{24-26}$

\section{Primera respiración al nacer y el momento en que se pinza el cordón umbilical}

Varios estudios demostraron que el tiempo en que se pinza el cordón tiene gran trascendencia sobre el momento en que se produce la primera respiración y en otros importantes aspectos de la adaptación fisiológica al nacer. Bhatt et al., en una investigación realizada en ovejas, ${ }^{27}$ estudiaron la influencia del clampeo demorado del cordón en la ventilación y la estabilidad hemodinámica cardiovascular y cerebral. Sus resultados demostraron que, si el clampeo se demoraba, al menos, hasta después de la primera respiración, mejoraba marcadamente la función cardiovascular, debido al aumento del flujo sanguíneo pulmonar en el RN y la consecuente estabilidad hemodinámica cerebral en los momentos claves de la transición posnatal. Asimismo, estos resultados mostraron que evitar el pinzamiento del cordón hasta después de la ventilación producía una mejor adaptación del $\mathrm{RN}$, uno de los beneficios más importantes del clampeo demorado del cordón.

El conocer estos mecanismos nos señala que, cuando el neonato está cianótico al nacer, no debemos clampear el cordón rápidamente, práctica errónea y muy frecuente. Con esta acción, solo lograremos empeorar la condición clínica, debido a que se produce marcada restricción del gasto cardíaco, que lleva al incremento de

FIGURA 1. Pasaje del hierro por el cordón umbilical

Miligramos de hierro que recibe el niño según tiempos de clampeo del cordón

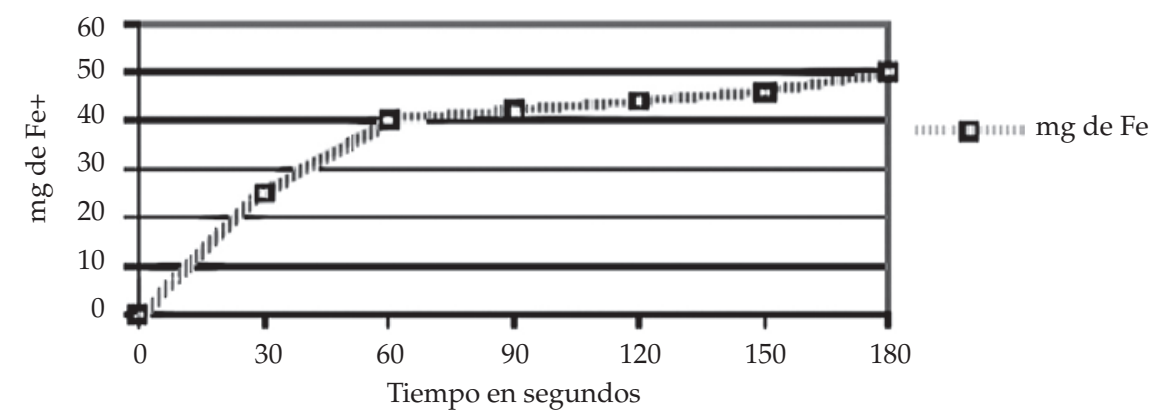


la hipoxia. Esto es así porque el aumento del gasto cardíaco es esencial para contrarrestar los efectos de la hipoxemia, y, por lo tanto, al limitar o impedir su aumento, se expone al bebé a la hipoxia e isquemia.

Un estudio desarrollado en África, en una extensa cohorte de $15563 \mathrm{RN},{ }^{28}$ evaluó la relación entre el tiempo de clampeo del cordón y el comienzo espontáneo de la primera respiración y la evolución en las primeras 24 horas de vida. Los resultados mostraron que el riesgo de muerte o admisión posalta fueron más altos cuando el clampeo del cordón se efectuaba antes de la primera respiración y esos riesgos disminuían en un $20 \%$ por cada 10 segundos de demora en pinzar el cordón.

Diversos artículos de actualización reafirman los beneficios del clampeo demorado por sobre el clampeo inmediato y exploran los diversos aspectos que pueden explicar la importancia de los mecanismos beneficiosos..$^{29-30}$

Hooper et al., ${ }^{31}$ enfatizan que el clampeo inmediato limita marcadamente el retorno venoso en ausencia de la respiración y eso reduce el gasto cardíaco, que permanece muy disminuido hasta que el RN comienza a respirar. Cuando así ocurre, la aeración del pulmón produce un marcado aumento de la circulación pulmonar, que incrementa el gasto cardíaco rápidamente, que se refleja por el franco aumento de la frecuencia cardíaca del bebé. Cabe destacar que, en la vida fetal, la fuente de la precarga para el ventrículo izquierdo proviene de la circulación de sangre desde la placenta y lo mismo se reproduce al nacer mientras la sangre de la placenta pasa al RN a través del cordón umbilical.

Reitero que, cuando los neonatos están apneicos y nos apuramos a clampear el cordón, cometemos un grave error porque, ante la restricción del gasto cardíaco, el RN permanecerá cianótico y con una circulación cerebral reducida.

Se insiste en que este aspecto reafirma que el gasto cardíaco es el mecanismo fisiológico más importante para evitar la hipoxia e isquemia en la circulación cerebral. Esto solo se logra mientras el cordón umbilical se mantenga sin pinzar y permita la transfusión placentaria. De esa forma, el RN evitará la restricción en la precarga y el gasto cardíaco, y tendrá una circulación más estable en la adaptación.

En publicaciones recientes, se remarca enfáticamente la importancia de conocer y aplicar la fisiología en el abordaje del tiempo de clampeo del cordón en la práctica clínica. ${ }^{32,33}$
Sin duda, la recomendación generalizada del clampeo inmediato del cordón, desde hace varias décadas y sin sustento alguno, ocasionaron innumerables perjuicios a los RN. En gran medida, esto ocurrió por desconocer que la transfusión placentaria producía una adecuada carga de volumen que prevenía el bajo gasto cardíaco, lo cual señala que ignoraban la fisiología en los procesos del pasaje de sangre desde la placenta al neonato.

En otro artículo reciente, la Dra. Niermeyer aborda un aspecto sumamente importante y de gran implicancia en la práctica cotidiana, que se refiere a la relación entre el tiempo de clampeo del cordón y la necesidad de reanimación en el RN. ${ }^{34}$

Cuando obstetras y neonatólogos acuerdan demorar el clampeo del cordón, solo lo hacen si el RN no requiere reanimación. Aquellos que necesitan resucitación son excluidos e inmediatamente se pinza el cordón umbilical. Sin embargo, esta medida no está justificada ni avalada por ningún estudio que haya demostrado que demorar el clampeo es perjudicial para la recuperación del neonato asfíctico. Por el contrario, hay una tendencia creciente a motivar que la reanimación se efectúe sin clampear el cordón en RNT. ${ }^{35,36} \mathrm{El}$ fundamento es similar a lo explicado, ya que también acá la fisiología juega un papel esencial al preservar la caída del gasto cardíaco.

Por lo tanto, no es adecuado efectuar de modo sistemático el clampeo del cordón antes de que comience la reanimación, ya que eso es nuevamente ignorar los conceptos fisiológicos de cómo transcurre la transición al nacer. Debemos considerar, como ya vimos, que lo apropiado es demorar el clampeo hasta un tiempo suficiente luego de una primera respiración tardía y lo mismo podríamos aplicar cuando necesitamos comenzar una reanimación.

Este aspecto se evaluó hace ya muchos años en un estudio experimental ${ }^{37}$ en monos Rhesus, al crear un modelo al nacer que les producía moderaba depresión, observaron que si pinzaban el cordón enseguida ocurría una marcada bradicardia acompañada por respiración irregular, jadeos y posterior apnea.

Estudios clínicos avalaron los posible beneficios de la transfusión placentaria en la reanimación en $\mathrm{RNT}^{35,36}$ pero aún son escasos los datos publicados en prematuros muy pequeños.

No obstante, en un estudio reciente, Kaempf et al. ${ }^{38}$ aportaron interesantes resultados al comparar la evolución clínica de 77 prematuros 
de muy bajo peso al nacer (MBPN) y 172 con bajo peso al nacer (BPN). Todos tenían menos de 35 semanas y el clampeo fue demorado hasta los 45 segundos versus un número igual de controles históricos nacidos durante el período inmediatamente anterior, cuando se practicaba el clampeo temprano. El puntaje de Apgar al minuto fue mayor en los prematuros de MBPN que recibieron clampeo demorado ( 7 vs. 5 , $\mathrm{p}<0,001)$ y también se observó una notable disminución del número de $\mathrm{RN}$ que requirieron oxígeno suplementario y medidas de ventilación en la recepción.

Sin embargo, es probable que, por ahora, no podamos recomendar la aplicación sistemática del clampeo demorado en todos los RNT que requieren reanimación, pero se puede poner en práctica, ya que las posibilidades de que sea beneficioso son altas al estar basadas en la fisiología, que, como siempre, sigue siendo segura.

\section{Las células madre y la transfusión placentaria}

La presencia de células madre en la sangre que proviene desde la placenta representa un aspecto de suma trascendencia, ya que juega un rol esencial, no solo en el neonato, sino también en niños y adultos.

El número de células madre es significativamente mayor en los prematuros extremos que en los RNT. Esto lo demostraron Haneline et al. ${ }^{39}$ que, además, observaron que la cantidad de células en la sangre del cordón era más elevada que en niños mayores e, incluso, que en la médula ósea de adultos. De acuerdo con estos hallazgos, el número de células madre en prematuros extremos sería el más elevado en la vida del ser humano.
En este proceso fisiológico, las células tienen múltiples funciones protectoras en el feto y luego del nacimiento. En el RN, se destacan mecanismos que regulan la maduración y el desarrollo en diversos órganos (en especial, el sistema nervioso central), las acciones antiinflamatorias y la reducción de varias enfermedades, tales como síndrome de dificultad respiratoria, anemia, sepsis, hemorragia intraventricular y leucomalacia periventricular. Entre otros trastornos, aún no plenamente confirmados, figuran retinopatía del prematuro y enterocolitis necrosante..$^{40}$

Aun cuando, al término, las células madre disminuyen, la cantidad con el clampeo demorado es más que suficiente, pero no es así cuando el clampeo es temprano, otro sólido argumento para no efectuar esta práctica.

Asimismo, la sangre del cordón contiene suficientes células madre hematopoyéticas para aplicarlas en trasplantes en niños, adolescentes y adultos,$^{41}$ una gran esperanza que crece firmemente. ${ }^{42,43}$

\section{Otros aspectos para tener en cuenta en el tiempo de clampeo del cordón umbilical}

\section{Niveles de bilirrubina plasmática}

Estudios de la década de 1970 observaron que los RN con clampeo demorado tenían valores de bilirrubinemia más elevados. ${ }^{5} \mathrm{El}$ aumento de la indicación de fototerapia también se ha descrito, pero no es unánime, aunque una revisión sistemática de ensayos clínicos observó que la indicación fue menor (en el límite) en neonatos con clampeo temprano. ${ }^{44}$

En el estudio que realizamos, ${ }^{9}$ no observamos ninguna diferencia entre los grupos, en los

TABLA 1. Niveles de bilirrubina plasmática entre las 36 y las 48 horas

\begin{tabular}{|c|c|c|c|c|c|c|c|c|c|}
\hline \multirow[t]{2}{*}{ Tratamiento } & \multicolumn{8}{|c|}{ Bilirrubinemia a las $36-48$ horas } & \multirow[b]{2}{*}{ Q75 } \\
\hline & $\mathbf{N}$ & Faltante & Min. & Máx. & Media & $\mathrm{DE}$ & Mediana & Q25 & \\
\hline Clampeo inmediato & 91 & 2 & 1 & 17,4 & 7,39 & 3,08 & 7,3 & 5,6 & 9 \\
\hline Clampeo al $1^{\mathrm{er}}$ minuto & 85 & 6 & 1 & 18,9 & 7,58 & 3,04 & 7,7 & 6,1 & 9 \\
\hline Clampeo al $3^{\text {er }}$ minuto & 90 & 2 & 1 & 14,6 & 6,99 & 2,98 & 7,2 & 5,0 & 9 \\
\hline Variable & \multicolumn{3}{|c|}{$\begin{array}{c}\text { Clampeo inmediato } \\
\mathrm{n} / \mathrm{N}(\%) \\
\end{array}$} & \multicolumn{3}{|c|}{$\begin{array}{c}\text { Clampeo al } 1^{\mathrm{er}} \text { minuto } \\
\mathrm{n} / \mathrm{N}(\%) \\
\end{array}$} & \multicolumn{3}{|c|}{$\begin{array}{c}\text { Clampeo al } 3^{\text {er }} \text { minuto } \\
\mathrm{n} / \mathrm{N}(\%) \\
\end{array}$} \\
\hline $\begin{array}{l}\text { Bilirrubina a las } 36-48 \text { horas } \\
\text { mayor de } 16 \mathrm{mg} / \mathrm{dL}\end{array}$ & \multicolumn{3}{|c|}{$2 / 91(2,20)$} & \multicolumn{3}{|c|}{$1 / 85(1,18)$} & \multicolumn{3}{|c|}{$0 / 90(0,0)$} \\
\hline
\end{tabular}

DE: desvío estándar. 
niveles de bilirrubina y en la indicación de luminoterapia (Tabla 1).

\section{Anemia al nacer y entre las 24 y las 48 horas}

La presencia de anemia temprana en nuestro estudio ${ }^{9}$ fue significativamente mayor en los RN con clampeo temprano del cordón (Tabla 2). La anemia al nacer es perjudicial porque los depósitos de Fe serán insuficientes tempranamente, y aumentará la presencia de anemia ferropénica, con sus posibles riesgos.

Es importante señalar que un análisis de subgrupo posterior a la publicación de nuestro estudio mostró que todos los RN con anemia (hematocrito $<45 \%$ ) a las 24 y las 48 horas eran nacidos por cesárea con clampeo temprano del cordón..$^{45}$ Este dato tiene una enorme relevancia debido al altísimo porcentaje actual de cesáreas y nos reafirma que, en esa práctica, también debemos aplicar el clampeo demorado del cordón.

\section{El estrés oxidativo}

Es conocido que los radicales libres producen diversos perjuicios en ciertas situaciones, entre ellas, en el momento del parto, cuando se ha comprobado estrés oxidativo en la madre, pero, en especial, en el RN. Probablemente, entre otros factores, el súbito pasaje del medio uterino con hipoxia fisiológica al brusco aumento de la presión arterial de oxígeno $\left(\mathrm{pO}_{2}\right)$ al nacer podría liberar radicales libres altamente tóxicos en el neonato. Para evitar daños en los tejidos, desde los primeros minutos posteriores al nacimiento, es necesario que actúen los sistemas antioxidantes para paliar los efectos perjudiciales del estrés oxidativo.

Hasta hace poco, no se tenía en cuenta que estos mecanismos pudiesen tener alguna relación con el tiempo de clampeo del cordón umbilical. En 2014, se publicó, en Pediatrics, ${ }^{46}$ el primer estudio que evaluó la influencia del clampeo temprano (10 segundos) versus demorado (2 minutos) sobre el estrés oxidativo. En madres sanas, parto vaginal y RNT sanos con Apgar $\geq 7$ al $1^{\text {er }}$ y $5^{\text {to }}$ minuto, se midieron, en el plasma de los neonatos, varios parámetros inflamatorios. Los resultados fueron contundentes al demostrar que todos los elementos protectores medidos (catalasa eritrocítica, superóxido dismutasa, factor de necrosis tumoral receptor ii, etc.) fueron significativamente más elevados en RN con clampeo demorado.

Aunque serán necesarios otros estudios aleatorizados, se abre un muy interesante terreno de investigación que aportará elementos importantes al decidir el tiempo de clampeo del cordón.

\section{El neurodesarrollo a largo plazo}

Hay muy pocos estudios que hayan evaluado la influencia del tiempo de clampeo del cordón en el neurodesarrollo a largo plazo, tanto en RNT como en prematuros. Recientemente, se publicaron estudios aleatorizados que evaluaron el neurodesarrollo al año ${ }^{47}$ y a los cuatro años, ${ }^{48}$ en una misma cohorte de niños nacidos a término con clampeo temprano (primeros 10 segundos) vs. demorado (3 minutos). La evaluación al año no mostró diferencias, aun cuando hubo una tendencia a mejores resultados en varones con clampeo demorado. La evaluación a los cuatro años es la primera investigación clínica sobre el neurodesarrollo en la edad preescolar. No observaron diferencias globales en el test de Wechsler Preschool and Primary Scale of Intelligene (WPPSI-III), pero los varones con clampeo demorado tuvieron un puntaje significativamente más alto que aquellos con clampeo temprano en el cociente de velocidad del procesamiento y también presentaron diferencias significativas en obtener mejores puntajes en las pruebas de

TABLA 2. Hematocrito central en las primeras 48 horas

\begin{tabular}{lccc}
\hline & Clampeo temprano & Clampeo al 1 $\mathbf{1}^{\text {er }}$ minuto & Clampeo al 3 \\
& $\mathbf{n = 9 0}$ & $\mathbf{n}=\mathbf{9 0}$ & $\mathbf{n}=\mathbf{9 2}$ \\
\hline Hematocrito $6 \mathrm{~h}<45 \%(\mathrm{n} \%)^{\mathbf{1}}$ & $8(8,9)$ & $1(1,1)$ & $0(0,0)$ \\
Hematocrito $24-48 \mathrm{~h}<45 \%(\mathrm{n} \%)^{\mathbf{2}}$ & $15(16,9)$ & $2(2,3)$ & $3(3,3)$ \\
\hline
\end{tabular}

${ }_{1}$ Clampeo al $1^{\text {er }}$ minuto vs. clampeo temprano: RR 0,3 (IC 95\%: 0,02-0,8); P <0,034.

${ }^{1}$ Clampeo al $3^{\text {er }}$ minuto vs. clampeo temprano: RR 0,06 (IC 95\%: 0,006-0,6); P <0,003.

${ }^{2}$ Clampeo al $1^{\text {er }}$ minuto vs. clampeo temprano: RR 0,13 (IC 95\%: 0,035-0,50); P <0,0014.

${ }^{2}$ Clampeo al $3^{\text {er }}$ minuto vs. clampeo temprano: RR 0,20 (IC 95\%: 0,06-0,61); P <0,0027.

Ceriani Cernadas JM, Carroli G, et al. Pediatrics 2006;117:e779-e786. 
movimientos, en el dominio del motor fino y en los dominios personales-sociales, en el test Ages and Stages Questionnaire (ASQ).

Cabe resaltar que los mejores resultados observados fueron en niños de muy bajo riesgo nacidos en un país sumamente desarrollado (Suecia). Esto es para tener en cuenta, ya que los efectos beneficiosos del clampeo demorado en el neurodesarrollo podrían tener mayor impacto en poblaciones desprotegidas con bajo nivel social y escasa educación. Aunque se ha publicado un estudio reciente, ${ }^{49}$ serán necesarias nuevas investigaciones acerca de los efectos del clampeo demorado a largo plazo.

\section{Reflexiones finales}

De acuerdo con todos los aspectos señalados, no hay dudas de que el clampeo demorado del cordón debe ser en los RNT o cerca del término el estándar de cuidado por las sólidas evidencias que lo apoyan.

A nuestro entender, es sumamente difícil encontrar, en la medicina, una práctica tan simple y sin costo alguno que tenga tan grandes beneficios a corto y largo plazo, con solo respetar los mecanismos fisiológicos y esperar unos pocos minutos.

Es necesario que los neonatólogos y pediatras tengamos en cuenta los ponderables efectos del clampeo demorado y breguemos por un mejor cumplimiento de esta práctica en la recepción de los RN.

Asimismo, deseamos señalar una reflexión que incluí en un comentario hace varios años, ${ }^{50}$ que no ha perdido vigencia y puede ayudar a comprender mejor por qué la práctica del clampeo demorado es la que se debe realizar:

Los beneficios hallados con el pinzamiento del cordón umbilical no antes de los 2 a 3 minutos después del nacimiento son producidos por hechos fisiológicos, por lo cual no podemos considerarlo realmente como un tratamiento, sino como una práctica que respeta los mecanismos fisiológicos naturales. Esos beneficios son principalmente el producto de permitir que el recién nacido reciba el volumen de sangre que le corresponde. Es decir, la transfusión placentaria es una inevitable consecuencia fisiológica en los primeros minutos de vida que proviene de la redistribución de la sangre entre la placenta y el neonato.

Por lo tanto, esto nos debe llevar a reflexionar acerca de conductas o prácticas equivocadas en el tiempo del clampeo, debido a que muchos desconocen que existe un fundamento fisiológico irrefutable.

Sin ninguna duda, la naturaleza ha determinado que el volumen de sangre de la placenta le pertenece en su gran mayoría al recién nacido, entonces, ¿por qué privarlo de que lo reciba?

\section{REFERENCIAS}

1. Darwin E. Zoonomia: or, The laws of organic life. Londres: Johnson; 1801.Págs.302.

2. Budin P. A quel moment doit-on pratiquer la ligature du cordon ombilical? Progr Med [Paris] 1875;3:765-7.

3. AGSP. 100 years on, and still no answer. Pediatrics 1975;55(6):813.

4. Usher R, Shephard M, Lind J. The blood volume of the newborn infant and placental transfusion. Acta Paediatr 1963;52:497-512.

5. Saigal S, O’Neill A, Surainder Y, Chua LB, et al. Placental transfusion and hyperbilirubinemia in the premature. Pediatrics 1972;49(3):406-19.

6. Yao AC, Lind J. Placental transfusion. Am J Dis Child 1974;127(1):128-41.

7. Saigal S, Usher RH. Symptomatic neonatal plethora. Biol Neonate 1977;32(1-2):62-72.

8. Linderkamp O, Versmold HT, Fendel H, Reigel KP, et al. Association of neonatal respiratory distress with birth asphyxia and deficiency of red cell mass in premature infants. Eur J Pediatr 1978;129(3):167-73.

9. Ceriani Cernadas JM, Carroli G, Pellegrini L, Otaño L, et al. The effect of timing of cord clamping on neonatal venous hematocrit values and clinical outcome at term: a randomized, controlled trial. Pediatrics 2006;117(4):e779-86.

10. Hutton EK, Hassan ES. Late vs Early Clamping of the Umbilical Cord in Full-term Neonates Systematic Review and Meta-analysis of Controlled Trials. JAMA 2007;297(11):1241-52.

11. McDonald SJ, Middleton P. Effect of timing of umbilical cord clamping of term infants on maternal and neonatal outcomes. Cochrane Database Syst Rev 2008;2(2):CD004074.

12. Ceriani Cernadas JM, Carroli G, Pellegrini L, Ferreira M, et al. Efecto del clampeo demorado del cordón umbilical en la ferritina sérica a los seis meses de vida. Estudio clínico controlado aleatorizado. Arch Argent Pediatr 2010;108(3):201-8.

13. Chaparro CM, Neufeld LM, Tena Alavez G, Eguia-Liz CR, ET al. Effect of timing of umbilical cord clamping on iron status in Mexican infants: a randomised controlled trial. Lancet 2006;367(9527):1997-2004.

14. Van Rheenen P, Gruschke S, Brabin BJ. Delayed umbilical cord clamping for reducing anaemia in LBW infants implications for developing countries. Ann Trop Paediatr 2006;26(3):157-67.

15. Van Rheenen P, Brabin BJ. Late umbilical cord clamping as an intervention for reducing iron deficiency anaemia in term infants in developing and industrialised countries. Ann Trop Paediatr 2004;24(1):3-16.

16. Gupta R, Ramji S. Effect of delayed cord clamping on iron stores in infants born to anemic mothers: a randomized controlled trial. Indian Pediatr 2002;39(2):130-5.

17. Linderkamp O. Placental transfusion: determinants and effects. Clin Perinatol 1982;9(3):559-92.

18. Pisacane A. Neonatal prevention of iron deficiency: placental transfusion is a cheap and physiological solution. BMJ 1996;312:136.

19. Geethanath RM, Ramji S, Thirupuram S, Rao YN. Effect of timing of cord clamping on the iron status of infants at 3 months. Indian Pediatr 1997;34(2):103-6.

20. Grajeda R, Perez-Escamilla R, Dewey K. Delayed clamping of the umbilical cord improves hematologic status of 
Guatemalan infants at 2 months of age. Am J Clin Nutr 1997;65(2):425-31.

21. Van Rheenen P, De Moor L, Eschbach S, de Grooth H, et al. Delayed cord clamping and haemoglobin levels in infancy: a randomised controlled trial in term babies. Trop Med Int Health 2007;12(5):603-16.

22. Andersson O,Hellström-Westas L, Andersson D, Domellöf M. Effect of delayed versus early umbilical cord clamping on neonatal outcomes and iron status at 4 months: a randomised controlled trial. BMJ 2011;343:d7157.

23. Kim AJH, Warren JB. OptimalTiming of Umbilical Cord Clamping: Is the Debate Settled? Part 2 of 2: Evidence in Preterm and Term Infants, Alternatives, and Unanswered Questions. NeoReviews 2015;16(5):e270-7.

24. Booth IW, Aukett MA. Iron deficiency anaemia in infancy and early childhood. Arch Dis Child 1997;76(6):549-54.

25. Carlson ES, Tkac I, Magid R, O'Connor MB, et al. Iron is essential for neuron development and memory function in mouse hippocampus. J Nutr 2009;139(4):672-9.

26. Szajewska H, Ruszczynski M, Chmielewska A. Effects of iron supplementation in nonanemic pregnant women, infants, and young children on the mental performance and psychomotor development of children: a systematic review of randomized controlled trials. Am J Clin Nutr 2010;91(6):1684-90.

27. Bhatt S, Alison BJ, Wallace EM, Crossley KJ, el al. Delaying cord clamping until ventilation onset improves cardiovascular function at birth in preterm lambs. J Physiol 2013;591(8):2113-26.

28. Ersdal HL, Linde J, Mduma E, Auestad B, et al. Neonatal outcome following cord clamping after onset of spontaneous respiration. Pediarics 2014;134(2);265-72.

29. Raju TNK. Timing of umbilical cord clamping after birth for optimizing placental transfusion. Curr Opin Pediatr 2013;25(2):180-7.

30. Duley L, Batey N. Optimal timing of umbilical cord clamping for term and preterm babies. Early Hum Dev 2013;89(11):905-8.

31. Hooper SB, Polglase GR, te Pas AB. A physiological approach to the timing of umbilical cord clamping at birth. Arch Dis Child Fetal Neonatal Ed 2015;100(4):F355-60.

32. Kluckow M, Hooper SB. Using physiology to guide time to cord clamping. Semin Fetal Neonatal Med 2015:20(4):225-31.

33. BoereI, Roest AA, WallaceE, Ten Harkel AD, etal.Umbilical blood flow patterns directly after birth before delayed cord clamping. Archs Dis Child Fetal Neonatal Ed 2015;100(2): F121-5.

34. Niermeyer S, Velaphi S. Promoting physiologic transition at birth: Re-examining resuscitation and the timing of cord clamping. Semin Fetal Neonatal Med 2013;18(6):385-92.

35. Hutchon D. Evolution of neonatal resuscitation with intact placental circulation. Clin Pract 2014;10(2):e58-61.
36. Perlman J, Kattwinkel J, Wyllie J, Guinsburg R, et al. Neonatal resuscitation: in pursuit of evidence gaps in knowledge. Resuscitation 2012;83(5):545-50.

37. Dawes GS, Jacobson HN, Mott JC, Shelley HJ, et al. The treatment of asphyxiated, mature foetal lambs and rhesus monkeys with intravenous glucose and sodiun carbonate. J Physiol 1963;169:167-84.

38. Kaempf JW, Tomlinson MW, Kaempf AJ, Wu Y, et al. Delayed umbilical cord clamping in premature neonates. Obstet Gynecol 2012;120(2 Pt 1):325-30.

39. Haneline LS, Marshall KP, Clapp DW. The highest concentration of primitive hematopoietic progenitor cells in cord blood is found in extremely premature infants. Pediatr Res 1996;39(5):820-5.

40. Lawton C, Acosta S, Watson N, González-Portillo C, et al. Enhancing endogenous stem cells in the newborn via delayed umbilical cord clamping. Neural Regen Res 2015;10(9):1359-62.

41. Tolosa JN, Park DH, Eve DJ, Klasko SK, et al. Mankind's first natural stem cell transplant. J Cell Mol Med 2010;14(3):488-95.

42. Chakraborty SK, Banu LA, Rahman MF, PaulS. Cord blood stem cells - a dream for future medicine. Mymensingh Med J 2014;23(3):614-20.

43. Milano F, Gooley T, Wood B, Woolfrey A, et al. Cord-Blood Transplantation in Patients with Minimal Residual Disease. N Engl J Med 2016;375(10):944-53.

44. McDonald SJ, Middleton P, Dowswell T, Morris PS. Effect of timing of umbilical cord clamping of term infants on maternal and neonatal outcomes. Cochrane Database Syst Rev 2013;(7):CD004074.

45. Ceriani Cernadas JM, Carroli G. Lether to the Editor. Pediatrics 2006;118:1317-9.

46. Díaz-Castro J, FloridoJ, Kajarabille N, Garrido-Sánchez M, et al. The Timing of Cord Clamping and Oxidative Stress in Term Newborns. Pediatrics 2014;134(2):257-64.

47. Andersson O, Domellöf M, Andersson D, Hellström-Westas L. Effect of Delayed vs Early Umbilical Cord Clamping on Iron Status and Neurodevelopment at Age 12 Months A Randomized Clinical Trial. JAMA Pediatr 2014;168(6):547-54.

48. Andersson O, Lindquist B, Lindgren M, Stjernqvist K, et al. Effect of Delayed Cord Clamping on Neurodevelopment at 4 years of age: A Randomized Clinical Trial. JAMA Pediatr 2015;169(7):631-8.

49. RabeH,Erickson-Owens DA, Mercer JS. Long-term Followup of Placental Transfusion in Full-term Infants. JAMA Pediatr 2015;169(7):623-4.

50. Ceriani Cernadas JM, Durán P. Commentary, Cochrane review: early versus delayed umbilical cord clamping in preterm infants. WHO Reproductive Health Library, 2006. [Acceso: 19 de octubre de 2016]. Disponible en: http:// apps.who.int/rhl/pregnancy_childbirth/childbirth/3rd_ stage/jccom/en/ 